\title{
Non-Curative Endoscopic Resection for Superficial Esophageal Cancer
}

\author{
Eun Hye Kim and Jun Chul Park \\ Division of Gastroenterology, Department of Internal Medicine, Severance Hospital, Yonsei University College of Medicine, Seoul, Korea
}

See "Long-Term Survival and Tumor Recurrence in Patients with Superficial Esophageal Cancer after Complete Non-Curative Endoscopic Resection: A Single-Center Case Series” by Ji Wan Lee, Charles J. Cho, Do Hoon Kim, et al., on page 470-477.

The incidence of esophageal cancer is increasing, and the disease prognosis is not good. ${ }^{1,2}$ Endoscopic mucosal resection and endoscopic submucosal dissection (ESD) are used for the management of esophageal squamous cell carcinomas (SCCs). ${ }^{3}$ Endoscopic resection can maintain esophageal integrity and avoid morbidity and mortality in esophagectomy. However, endoscopic resection is limited in that it cannot approach lymph node metastases, although lymph node status is an important prognostic factor in esophageal cancer.

Multiple studies have shown that SCCs limited to the epithelial layer (m1) and lamina propria mucosa (m2) without lymphovascular involvement have a low risk of lymph node metastasis. ${ }^{4}$ If esophageal lesions are confined to $\mathrm{m} 1$ or $\mathrm{m} 2$, endoscopic resection may be considered. ${ }^{5,6}$ However, if SCCs invade the muscularis mucosa (m3) or the upper $200 \mu \mathrm{m}$ of the submucosa (sm1), the probability of lymph node metastasis is increased. The Japan Esophageal Society has suggested absolute indications for endoscopic resection of lesions confined to the $\mathrm{m} 1$ or $\mathrm{m} 2$, and relative indications for endoscopic resection of lesions confined to the $\mathrm{m} 3$ or $\mathrm{sm} 1 .{ }^{8}$ The European

Received: August 13, 2018 Revised: September 10, 2018

Accepted: September 12, 2018

Correspondence: Jun Chul Park

Division of Gastroenterology, Department of Internal Medicine, Severance Hospital, Yonsei University College of Medicine, 50-1 Yonsei-ro, Seodaemun-gu, Seoul 03722, Korea

Tel: +82-2-2228-2272, Fax: +82-2-393-6884, E-mail: JUNCHUL75@yuhs.ac ORCID: https://orcid.org/0000-0001-8018-0010

(c) This is an Open Access article distributed under the terms of the Creative Commons Attribution Non-Commercial License (http://creativecommons.org/ licenses/by-nc/3.0) which permits unrestricted non-commercial use, distribution, and reproduction in any medium, provided the original work is properly cited.
Society of Gastrointestinal Endoscopy has also recommended endoscopic resection of superficial esophageal SCCs classified as $\mathrm{m} 1$ or $\mathrm{m} 2$ at very low risk of lymph node metastasis. ${ }^{9}$ SCCs that invade deeper than the upper $200 \mu \mathrm{m}$ of the submucosa carried a high risk of lymph node metastasis and should not be considered for endoscopic resection. ${ }^{7,10}$

In a recent Japanese study, the authors reported that during a 3-year follow-up period, the relapse-free survival rates of patients who received $(n=26)$ or did not receive additional treatments $(n=17)$ for superficial esophageal cancer with submucosal invasion were $88 \%$ and $64 \%$, respectively, and the result was significantly different (95\% confidence interval, 0.45-0.76; $p=0.04) .{ }^{11}$ However, they included patients who underwent non-complete resection and had a positive resection margin. In a recent issue of Clinical Endoscopy, Lee et al. analyzed long-term survival and tumor recurrence in patients with superficial esophageal cancer after complete non-curative endoscopic resection. ${ }^{12}$ In their study, non-curative resection was defined as the presence of submucosal and/or lymphovascular invasion on endoscopic resection pathology. Patients who were margin positive were excluded from the non-complete resection group. The 5-year overall survival rate was $72.9 \%$ in the close observation group $(n=10)$ and $82.1 \%$ in the additional treatment group $(n=14 ; p=0.958){ }^{12}$ In addition, they reported similar 5-year cumulative incidences of primary esophageal cancer recurrence $(10.0 \%$ vs. $16.4 \%, p=0.558)$ and metachronous esophageal cancer recurrence ( $16.7 \%$ vs. $26.7 \%$, $p=0.667$ ) between the two groups. ${ }^{12}$ Given these results, the authors concluded that if the esophageal lesion was resected completely, even though it was non-curatively resected, close 
observation might be an option for patients who are medically unfit to receive additional therapy.

The possibility of lymph node metastasis and tumor recurrence, however, should remain a concern when observing patients with non-curatively resected esophageal cancer. The risk of lymph node metastasis could be increased according to the depth of invasion of esophageal cancer. It increases to up to approximately $20 \%$ for lesions invading the m3, up to approximately $40 \%-50 \%$ for lesions invading the sm1, and up to $54 \%$ for deeper lesions (sm2). ${ }^{9}$ A recent study that analyzed 43 patients with T1 esophageal SCC reported lymph node metastasis rates and no lymph node metastasis in $\mathrm{m} 1$ and $\mathrm{m} 2$ esophageal lesions. The lymph node metastasis rates increased according to the depth of invasion. ${ }^{13}$ No hematologic recurrence was observed in $\mathrm{m} 1, \mathrm{~m} 2, \mathrm{~m} 3$, and $\mathrm{sm} 1$ lesions. However, the incidence rate of hematologic recurrence were $9 \%$ and $13 \%$ in the sm 2 and $\mathrm{sm} 3$ cases, respectively. ${ }^{13}$ They discerned that if the tumor depth was greater than the $\mathrm{sm} 2$, patients showed higher rates of lymph node involvement and hematological recurrence, such as liver or lung metastases. ${ }^{13}$ Meanwhile, the 5-year disease-specific survival rates were $100 \%$ in mucosal lesions. However, the survival rates were decreased according to the depth of tumor invasion in submucosal cancer as follows: $90.0 \%$ (sm1), 78.8\% (sm2), and 68.6\% (sm3). ${ }^{13}$ In another study, Yamashina et al. investigated the survival and metastasis rates after endoscopic resection of esophageal carcinoma. ${ }^{14}$ The 5-year overall survival rates of the patients were decreased according to the depth of tumor invasion as follows: approximately $90 \%$ for $\mathrm{m} 1 / \mathrm{m} 2,71 \%$ for $\mathrm{m} 3$, and $70 \%$ for submucosal cancer $(p=0.007){ }^{14}$ In a multivariate analysis, the depth of tumor invasion and age were independent factors of survival; the hazard ratio was 3.6 for muscularis mucosal cancer and 3.2 for the submucosal cancer, as compared with $\mathrm{ml} /$ $\mathrm{m} 2$ cancer, and 1.07 per year of age. ${ }^{14}$ The cumulative 5-year metastasis rates were increased according to tumor depth as follows: $0.4 \%$ for $\mathrm{m} 1 / \mathrm{m} 2$, and approximately $8 \%$ for $\mathrm{m} 3$, $7 \%$ for sm 1 , and $36 \%$ for $\mathrm{sm} 2(p<0.001) .{ }^{14}$ In a multivariate analysis, the depth of invasion was an independent risk factor of metastasis, and the hazard ratio was 13.1 for muscularis mucosal cancer, 40.2 for SM1, and 196.3 for SM2, as compared with $\mathrm{m} 1 / \mathrm{m} 2$ cancer. $^{14}$

With non-curative resection of esophageal cancer, no additional treatment has been established yet. However, chemoradiotherapy (CRT) can be considered in non-curative resection cases. Kawaguchi et al. reported the pros and cons of ESD in addition to CRT for superficial esophageal cancer treatment. ${ }^{15}$ In this study, they divided the enrolled patients to a "CRT after ESD” group (ESD-CRT group) and a CRT-only group (dCRT group). The patients in the ESD-CRT group were defined as those with lesions invading the muscularis mucosa $(\mathrm{m} 3)$ or submucosal layer (T1b) with vascular invasion. The patients in the dCRT group were defined as those who received definitive CRT only. Sixteen patients were enrolled in the ESDCRT group; and 31, in dCRT group. ${ }^{15}$ The results showed that 6 patients in the dCRT group had a local recurrence (19\%), whereas none of the patients in the ESD-CRT group had a local recurrence $(p=0.029){ }^{15}$ Hamada et al. also reported that endoscopic resection followed by CRT attained survival rates (5-year overall survival rate, 75\%) comparable with those with esophagectomy or definitive CRT. ${ }^{16}$ In their study, cancers without lymphovascular involvement showed favorable outcomes. ${ }^{16}$ None of the 30 patients without lymphovascular involvement had a metastatic recurrence, whereas 6 of the 36 patients with lymphovascular involvement showed a metastatic recurrence $(p=0.0098) .{ }^{16}$ These results suggest that CRT after ESD could be an effective method for non-curatively resected lesions. However, further studies would be needed to demonstrate the efficacy of CRT after non-curative resection of esophageal cancer.

It is meaningful that Lee et al. reported the long-term outcomes of treatment and observation groups after non-curative resection of esophageal cancer among Korean patients. ${ }^{12}$ It is particularly meaningful that their study showed close observation to be a potential option for patients who have undergone a complete non-curative resection and are unable to receive additional therapy considering the high mortality associated with esophagectomy. However, more well-designed studies with larger sample sizes are needed to definitively determine which patients should be eligible for observation without additional therapy.

Conflicts of Interest

The authors have no financial conflicts of interest.

\section{REFERENCES}

1. Mariette C, Piessen G, Triboulet JP. Therapeutic strategies in oesophageal carcinoma: role of surgery and other modalities. Lancet Oncol 2007;8:545-553.

2. Koizumi S, Jin M, Matsuhashi T, et al. Salvage endoscopic submucosal dissection for the esophagus-localized recurrence of esophageal squamous cell cancer after definitive chemoradiotherapy. Gastrointest Endosc 2014;79:348-353.

3. Sgourakis G, Gockel I, Lang H. Endoscopic and surgical resection of Tla/T1b esophageal neoplasms: a systematic review. World J Gastroenterol 2013;19:1424-1437.

4. Hammad H, Kaltenbach T, Soetikno R. Endoscopic submucosal dissection for malignant esophageal lesions. Curr Gastroenterol Rep 2014;16:386.

5. Araki K, Ohno S, Egashira A, Saeki H, Kawaguchi H, Sugimachi K. Pathologic features of superficial esophageal squamous cell carcinoma with lymph node and distal metastasis. Cancer 2002;94:570-575.

6. Kim DU, Lee JH, Min BH, et al. Risk factors of lymph node metastasis 
in T1 esophageal squamous cell carcinoma. J Gastroenterol Hepatol 2008;23:619-625.

7. Oyama T, Tomori A, Hotta K, et al. Endoscopic submucosal dissection of early esophageal cancer. Clin Gastroenterol Hepatol 2005;3(7 Suppl 1):S67-S70.

8. Japan Esophageal Society. Japanese classification of esophageal cancer, 11th edition: part I. Esophagus 2017;14:1-36

9. Pimentel-Nunes P, Dinis-Ribeiro M, Ponchon T, et al. Endoscopic submucosal dissection: European Society of Gastrointestinal Endoscopy (ESGE) guideline. Endoscopy 2015;47:829-854.

10. Gockel I, Sgourakis G, Lyros O, et al. Risk of lymph node metastasis in submucosal esophageal cancer: a review of surgically resected patients. Expert Rev Gastroenterol Hepatol 2011;5:371-384.

11. Ikeda A, Hoshi N, Yoshizaki T, et al. Endoscopic submucosal dissection (ESD) with additional therapy for superficial esophageal cancer with submucosal invasion. Intern Med 2015;54:2803-2813.

12. Lee JW, Cho CJ, Kim DH, et al. Long-term survival and tumor recur- rence in patients with superficial esophageal cancer after complete non-curative endoscopic resection: a single-center case series. Clin Endosc 2018;51:470-477.

13. Akutsu Y, Uesato M, Shuto K, et al. The overall prevalence of metastasis in T1 esophageal squamous cell carcinoma: a retrospective analysis of 295 patients. Ann Surg 2013;257:1032-1038.

14. Yamashina $T$, Ishihara $R$, Nagai $K$, et al. Long-term outcome and metastatic risk after endoscopic resection of superficial esophageal squamous cell carcinoma. Am J Gastroenterol 2013;108:544-551.

15. Kawaguchi G, Sasamoto R, Abe E, et al. The effectiveness of endoscopic submucosal dissection followed by chemoradiotherapy for superficial esophageal cancer. Radiat Oncol 2015;10:31.

16. Hamada K, Ishihara R, Yamasaki Y, et al. Efficacy and safety of endoscopic resection followed by chemoradiotherapy for superficial esophageal squamous cell carcinoma: a retrospective study. Clin Transl Gastroenterol 2017;8:e110. 